\title{
E-cadherin knockdown increases $\beta$-catenin reducing colorectal cancer chemosensitivity only in three-dimensional cultures
}

\author{
XI LIANG ${ }^{1,4}$, XUEQING XU ${ }^{4}$, FENGCHAO WANG ${ }^{2}$, XUEDAN CHEN $^{3}$, \\ NI LI ${ }^{1}$, CANCAN WANG ${ }^{1}$ and JIANMING HE ${ }^{1}$
}

${ }^{1}$ Department of Oncology and Southwest Cancer Center, Southwest Hospital, ${ }^{2}$ Institute of Combined Injury,

State Key Laboratory of Trauma, Burns and Combined Injury, ${ }^{3}$ Department of Medical Genetics,

Third Military Medical University, Chongqing 400038; ${ }^{4}$ Molecular Biology Center, State Key Laboratory of Trauma,

Burn, and Combined Injury, Daping Hospital, Third Military Medical University, Chongqing 400042, P.R. China

Received June 12,2015; Accepted August 11, 2015

DOI: $10.3892 /$ ijo.2015.3137

\begin{abstract}
Decreased expression of E-cadherin correlates with poor prognosis in colorectal cancer. Certain E-cadherin signaling cascades are triggered by intercellular force or binding to cadherins on adjacent cells. Three-dimensional (3D) cell cultures represent a better approximation of cell-cell adhesion in vivo than two-dimensional (2D) cultures. Here, we explored the role of E-cadherin in colorectal cancer chemosensitivity in $3 \mathrm{D}$ cultures. Cell-cell junctions, including tight junctions, gap junctions, intermediate junctions and desmosomes, were commonly found in 3D cultures. Knockdown of E-cadherin by lentiviral delivery of shRNA significantly reduced chemosensitivity to 5 -fluorouracil and irinotecan, increased $\beta$-catenin protein level in HCT116 3D cultures. However, these effects were not observed in 2D cultures. Knockdown of $\beta$-catenin significantly increased chemosensitivity to 5 -fluorouracil and irinotecan in HCT116 3D cultures and LoVo 3D cultures. 5-Fluorouracil activated p38, ERK1/2 and JNK1/2 in a time-dependent manner in HCT116 3D cultures. E-cadherin knockdown enhanced p-p38 and p-ERK1/2, except p-JNK1/2 in HCT116 3D cultures. Knockdown of $\beta$-catenin attenuated p-p38 and p-ERK1/2 in HCT116 3D cultures and LoVo 3D
\end{abstract}

Correspondence to: Dr Jianming He, Department of Oncology and Southwest Cancer Center, Southwest Hospital, Third Military Medical University, Shapingba, Chongqing 400038, P.R. China E-mail: hejm@tmmu.edu.cn

Abbreviations: 3D, three-dimensional; 2D, two-dimensional; Ecad, E-cadherin; sh, small hairpin; H\&E, hematoxylin and eosin; DAPI, 4',6-diamidino-2-phenylindole; MAPK, mitogen-activated protein kinase; WST assay, tetrazolium salt-based proliferation assay; 5-FU, 5-fluorouracil; CPT-11, irinotecan; ERK, extracellular-signalregulated kinase; JNK, c-Jun $\mathrm{N}$-terminal kinase; shEcad, knockdown E-cadherin by shRNA; sh $\beta$-catenin, knockdown $\beta$-catenin by shRNA; shcontrol, control shRNA targeted against green fluorescent protein; d, day; h, hour

Key words: colorectal cancer, E-cadherin, $\beta$-catenin, p38 mitogenactivated protein kinase, extracellular-signal-regulated kinase cultures. Inhibition of p-p38 or p-ERK1/2 in HCT116 3D cultures significantly increased chemosensitivity. Our results indicate E-cadherin knockdown increases $\beta$-catenin resulting in reduction of chemosensitivity only in $3 \mathrm{D}$ cultures, and $\beta$-catenin increasing the $\mathrm{p}-\mathrm{p} 38 / \mathrm{p}-\mathrm{ERK} 1 / 2$ is involved in this mechanism.

\section{Introduction}

Cells in vivo proliferate and survive under the influence of adjacent cells (1-3). Cell-cell adhesion is important for tumor cell biological behavior, including chemosensitivity $(1,2,4-7)$. Intercellular adhesion is not well recapitulated in two-dimensional (2D) cell cultures and this may explain, in part, the phenomenon that novel anticancer drugs often offer only a small improvement over older agents or even fail to offer improvement in clinical trials, though they exhibit exciting anticancer effect in vitro (1-6). Three-dimensional (3D) cell culture models represent a better approximation of solid tumor tissue microenvironment, including cell adhesion and chemosensitivity in vivo than $2 \mathrm{D}$ cultures (1-8).

E-cadherin (Ecad), a classic member of the cadherin family, is responsible for cell-cell adhesion (2,9-13). On the other hand, intercellular force and E-cadherin binding to cadherins on adjacent cells trigger certain E-cadherin signaling cascades $(9,10,12)$. Decreased expression of E-cadherin is strongly correlated with colorectal cancer, including prognosis $(14,15)$. Therefore, we explored the role of E-cadherin in colorectal cancer chemosensitivity using 3D cultures. E-cadherin knockdown significantly reduced chemosensitivity via increasing $\beta$-catenin in 3D cultures while these effects were not observed in $2 \mathrm{D}$ cultures.

\section{Materials and methods}

Cell lines and cell culture. Human colorectal cancer cell lines, HCT116, HT29, LoVo and human embryonic kidney 293T were obtained from the Cell Bank, Chinese Academy of Science.

$2 \mathrm{D}$ cultures were routinely grown and passaged as previously described $(4,16)$. In brief, cells were grown in McCoy's 5A 
(Gibco, Grand Island, NY, USA) (HCT116 and HT29), F12K (Gibco) (LoVo) or DMEM (293T) (Gibco) supplemented with $100 \mathrm{ml} / \mathrm{l}$ fetal bovine serum (Gibco), 100,000 IU/l penicillin, and $100 \mathrm{mg} / \mathrm{l}$ streptomycin (Gibco) under a humidified atmosphere of $5 \% \mathrm{CO}_{2}$ at $37^{\circ} \mathrm{C}$.

$3 \mathrm{D}$ cultures were prepared by using the liquid overlay technique as previously described $(2,4,17)$. In brief, exponentially-growing cancer cells were seeded into plates what were previously coated with $2 \%$ agarose. Plates were gently horizontally swirled $10 \mathrm{~min}$ every $6 \mathrm{~h}$ for the first $24 \mathrm{~h}$ on an orbital shaker in order to form multicellular spheroids. Cells were incubated under a humidified atmosphere of $5 \% \mathrm{CO}_{2}$ at $37^{\circ} \mathrm{C}$. Appropriate medium was refreshed every day.

Lentiviral delivery of shRNA. E-cadherin and $\beta$-catenin were knocked down through the use of lentiviral vector-mediated shRNA interference using The RNAi Consortium System (Open Biosystems, Inc., Huntsville, AL, USA) according to the manual, respectively (18). Sense sequences of shRNAs targeting specific genes are AAGATAGGAGTTCTCTGATGC (shEcad-1) or ATACCAGAACCTCGAACTATA (shEcad-2) for E-cadherin (18) and GCTTGGAATGAGACTGCTGAT for $\beta$-catenin (sh $\beta$-catenin) (18). Control shRNA (shcontrol) is targeted against green fluorescent protein and the sense sequence of shRNA is TACAACAGCCACAACGTCTAT. E-cadherin/ $\beta$-catenin-targeting shRNA-pLKO.1 vector or a control shRNA-pLKO.1 vector with the packaging plasmid pCMV-Dr8.91 and the enveloping plasmid pCMV-VSV-G were co-transfected into 293T cells with Lipofectamine ${ }^{\circledR} 2000$ (Invitrogen, Carlsbad, CA, USA) according to the manual. Virus-containing media was collected at 48 and $72 \mathrm{~h}$ posttransfection, and was filtered. Cells were infected with lentivirus encoding shRNA targeted specific genes or control lentivirus, respectively. Then, cells were selected using puromycin (Sigma-Aldrich, St. Louis, MO, USA). Knockdown efficiency was confirmed by western blotting.

Hematoxylin and eosin $(H \& E)$ staining. HCT116 3D cultures were collected in a $1.5 \mathrm{ml}$ Eppendorf tube. Following centrifugation $\left(100 \mathrm{~g}, 2 \mathrm{~min}, 4^{\circ} \mathrm{C}\right)$, the supernatant was discarded. The pellet was fixed in $4 \%$ paraformaldehyde for $30 \mathrm{~min}$. Following centrifugation (100 g, $3 \mathrm{~min}$ ), the pellet was placed in 100\% ethanol and xylene for $10 \mathrm{~min}$ at room temperature, and then in paraffin for $20 \mathrm{~min}$ at $65^{\circ} \mathrm{C}$, respectively. The sample was embedded in paraffin and the paraffin-embedded sample was sectioned at a thickness of $10 \mu \mathrm{m}$. Sample slides were routinely stained with H\&E.

Colorectal tumors were induced in C57BL/6 mice by azoxymethane (Sigma-Aldrich)-dextran sodium sulfate (MP Biomedicals, molecular weight 36,000-50,000 Da., Irvine, CA, USA) as previously described (19). Mice were sacrificed 20 weeks after azoxymethane treatment and colorectums were excised. Colorectal tumors were fixed in 10\% formalin/PBS and paraffin-embedded samples were sectioned at a thickness of $6 \mu \mathrm{m}$. Sample slides were routinely stained with H\&E. The study was approved by the Ethics Committee of Southwest Hospital.

Immunofluorescence staining. HCT116 cells were cultured as monolayer on cover slides for $72 \mathrm{~h}$. After fixation in
4\% paraformaldehyde for $30 \mathrm{~min}$, cells were incubated in $0.2 \%$ Triton X-100 in 2\% BSA/PBS for 30 min. Antibody of $\beta$-catenin (Cell Signaling Technology, Beverly, MA, USA) and Alexa Fluor 555 goat anti-rabbit (Invitrogen) were incubated for $2 \mathrm{~h}$ and $30 \mathrm{~min}$, respectively. 4',6-diamidino-2-phenylindole (DAPI) $(1 \mu \mathrm{g} / \mathrm{ml})$ (Sigma-Aldrich) was used for staining the nucleus of cells for $30 \mathrm{~min}$.

Immunohistochemical staining. HCT116 3D cultures were fixed in 4\% paraformaldehyde and OCT embedded samples were sectioned at a thickness of $10 \mu \mathrm{m}$. Immunohistochemistry was performed according to protocol of the SPlink Detection kits (ZSGB-Bio, Beijing, China) as previously described (16).

Preparation for transmission electron microscope slides. Sample slides were routinely prepared as previously described (4). In brief, 3D cultures were fixed in 2.5\% glutaraldehyde, and then in $1 \%$ osmium tetroxide. Samples were dehydrated by graded alcohol, ultrathin sectioned. Sections were stained with uranium acetate and lead citrate, and observed using a transmission electron microscope (TECNAI10, Philip, The Netherlands).

Preparation of cell lysates. Cells were lysed in RIPA buffer (50 mM Tris base, $150 \mathrm{mM} \mathrm{NaCl}, 1 \%$ Nonidet P-40, $0.25 \%$ Na-deoxycholate, $1 \mathrm{mM}$ EDTA) with protease inhibitors and phosphatase inhibitors ( $1 \mathrm{mM}$ PMSF, $5 \mu \mathrm{g} / \mathrm{ml}$ leupeptin, $2 \mu \mathrm{g} /$ ml pepstatin, $4 \mu \mathrm{g} / \mathrm{ml}$ aprotinin, $10 \mathrm{mM} \mathrm{NaF}, 1 \mathrm{mM} \mathrm{Na}_{3} \mathrm{VO}_{4}$, $10 \mathrm{mM} \beta$-glycerophosphate disodium salt pentahydrate) by incubating for $30 \mathrm{~min}$ on ice. Following centrifugation $\left(26,000 \mathrm{x} \mathrm{g}, 16 \mathrm{~min}, 4^{\circ} \mathrm{C}\right)$, the supernatant was collected as total cell protein (19).

Western blot analysis. Protein was resolved by SDS/PAGE and blotted on nitrocellulose membranes (Bio-Rad, Richmond, CA, USA) as previously described $(4,16,19)$. Nitrocellulose membranes were incubated with specific primary antibodies overnight. After incubating with secondary antibodies, immunoreactive proteins were visualized by the enhanced chemiluminescnet substrate (Thermo Scientific, Pittsburgh, PA, USA).

E-cadherin antibody was from Abcam Inc. (Cambridge, MA, USA). $\beta$-catenin antibody, phospho-mitogen-activated protein kinase (MAPK) Family Antibody Sampler kit, $\alpha$-tubulin antibody, $\beta$-actin antibody, GAPDH antibody, histone H3, HRP-linked secondary antibody were from Cell Signaling Technology.

Clonogenic assay. Clonogenic assay in vitro were routinely performed as previously described (20). In brief, HCT116 3D cultures were collected in $2 \mathrm{ml}$ medium. $0.2 \mathrm{ml}$ medium containing 3D cultures was taken into a $0.5 \mathrm{ml}$ Eppendorf tube. Following centrifugation (100 g, $5 \mathrm{~min}$ ), the pellet was detached by accutase and the cells number of the singlecell suspension were assayed. Then, the cells number of the 3D cultures was calculated. The same amount of HCT116 3D cultures was treated with $80 \mathrm{mg} / 15$-FU for $24 \mathrm{~h}$. Then, 3D cultures were detached by accutase and the same ratio of single-cell suspensions were seeded into 24 -well plates 
A

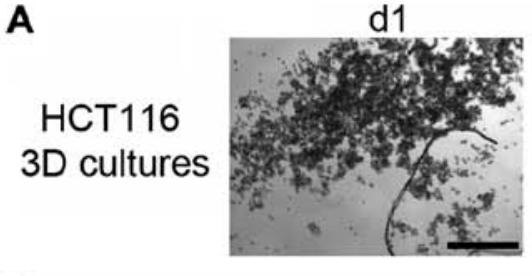

B

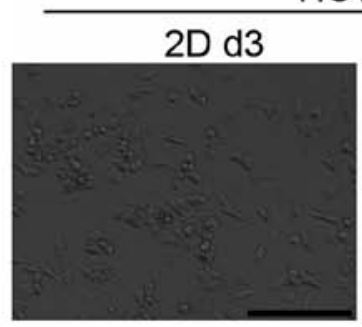

HCT116

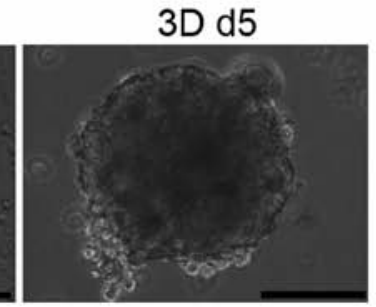

d2

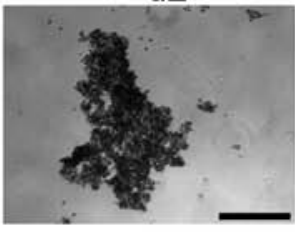

d3

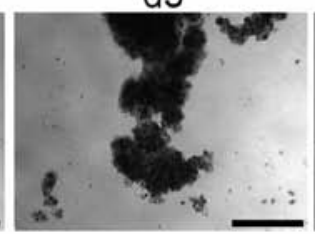

C
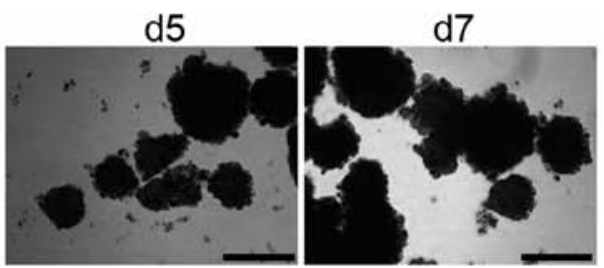

D

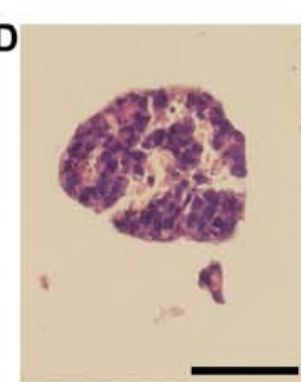

E
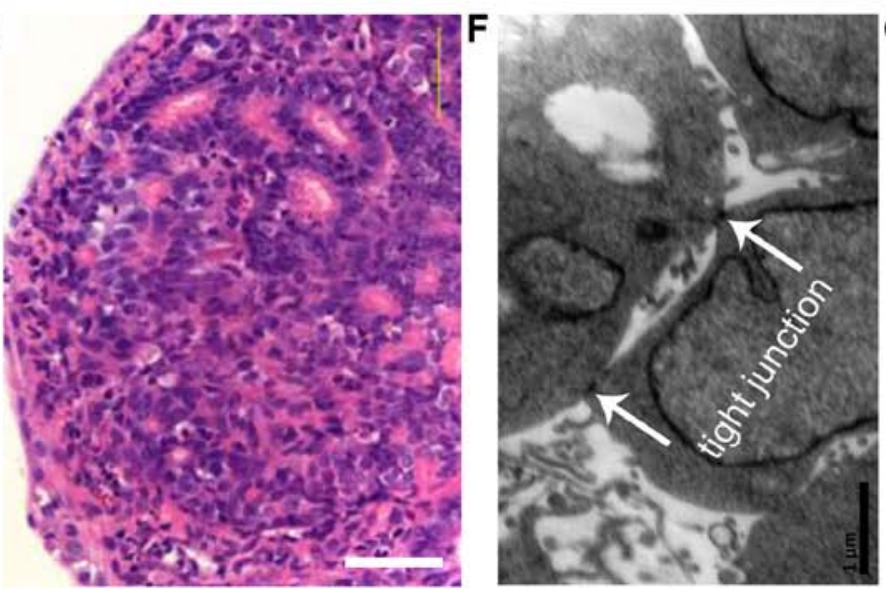

G
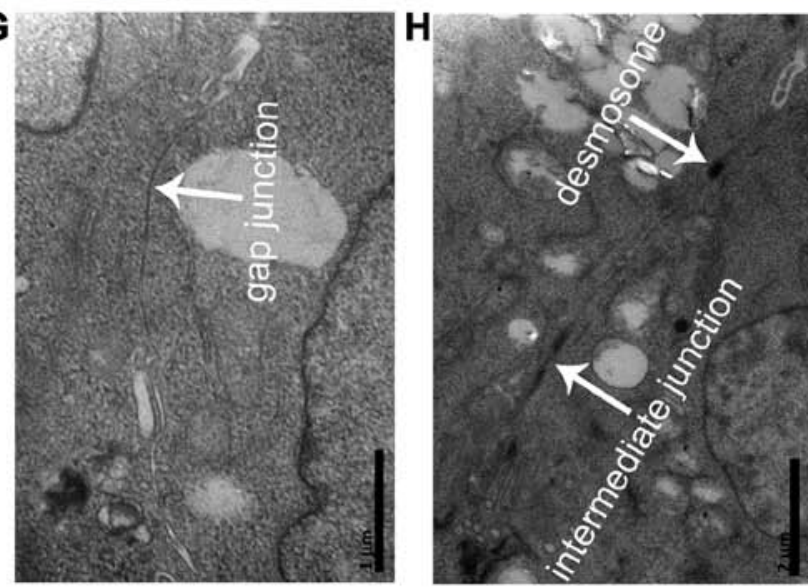

Figure 1. Intercellular adhesion in 3D cultures (multicellular spheroids). (A) HCT116 cells were cultured under 3D condition and were photographed at different time-points. Scale bars represent $400 \mu \mathrm{m}$. (B and C) HCT116 and HT29 were cultured under 2D condition for 3 days and 3D condition for 5 days, respectively. Scale bars represent $200 \mu \mathrm{m}$. (D) HCT116 3D cultures were stained with H\&E. The scale bar represents $50 \mu \mathrm{m}$. (E) Mouse colorectal cancer at avascular stage was stained with H\&E. The scale bar represents $50 \mu \mathrm{m}$. (F-H) Cell-cell junctions, including tight junctions (F) (the scale bar represents $1 \mu \mathrm{m}$ ), gap junctions (G) (the scale bar represents $1 \mu \mathrm{m}$ ), intermediate junctions and desmosomes (H) (the scale bar represents $2 \mu \mathrm{m}$ ) were found in most cells in 3D cultures using a transmission electron microscope (d, day).

duplicate. Cells were cultured at $37^{\circ} \mathrm{C}$ for 7 days, then stained with crystal violet.

WST assay for sensitivity to anticancer drugs. Cytotoxic activity in 2D cultures was determined by tetrazolium saltbased proliferation assay (WST assay) using the cell counting kit-8 (Dojindo Laboratories, Kumamoto, Japan) according to the manual as previously described $(4,21)$. In brief, HCT116 cells were cultured in 96-well plates as monolayer. Then, $10 \mu \mathrm{l}$ of a graded concentration of 5-fluorouracil (5-FU) or irinotecan (CPT-11) were added into each well and cultured for $24 \mathrm{~h}$. Control cultures received $10 \mu \mathrm{l}$ PBS only. Each contained 8 independent samples. After $24 \mathrm{~h}, 10 \mu \mathrm{l}$ of WST solution were added to each well and the plates were incubated for another $2 \mathrm{~h}$. Absorbance was measured at $450 \mathrm{~nm}$ using a microplate reader with reference wavelength of $650 \mathrm{~nm}$. Cell viability was measured and compared with that of control cells.

Cytotoxic activity in 3D cultures was determined by WST using the similar way to assay cytotoxic activity in 2D cultures as previously described $(4,17,21)$. Before incubating with WST,
$3 \mathrm{D}$ cultures were detached by accutase for $2 \mathrm{~min}$. Cell viability was measured and compared with that of 3D cultures treated with PBS.

Statistical analyses. The data shown represent the mean \pm standard error. Statistical differences between groups were analyzed by one-way ANOVA. $\mathrm{p}<0.05$ was considered statistically significant.

\section{Results}

Cell-cell adhesion in 3D cultures. When seeded under nonadhesive conditions, dispersed cells (HCT116 and HT29) aggregated automatically and formed 3D cultures (multicellular spheroids) within $24 \mathrm{~h}$. Cells adhered to each other loosely. Three days later, cells adhered tightly to other cells and 3D cultures could be hardly dispersed into single cells by pipetting (Fig. 1A-C).

To further analyze cell-cell adhesive systems, HCT116 3D cultures were stained with H\&E. 3D cultures consisted of layers of cells and the cells were packed tightly (Fig. 1D). These 
A

HCT116 2D
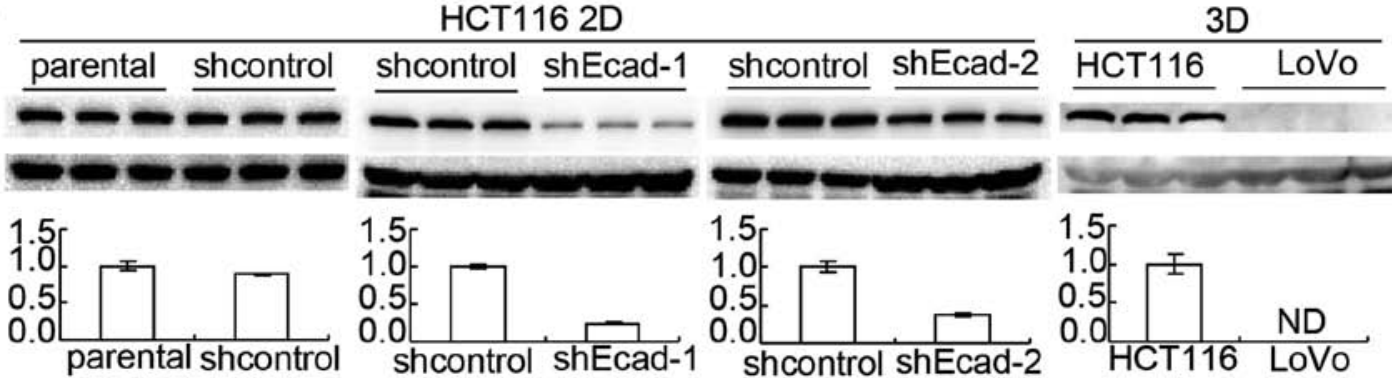

Ecad

$\beta$-actin
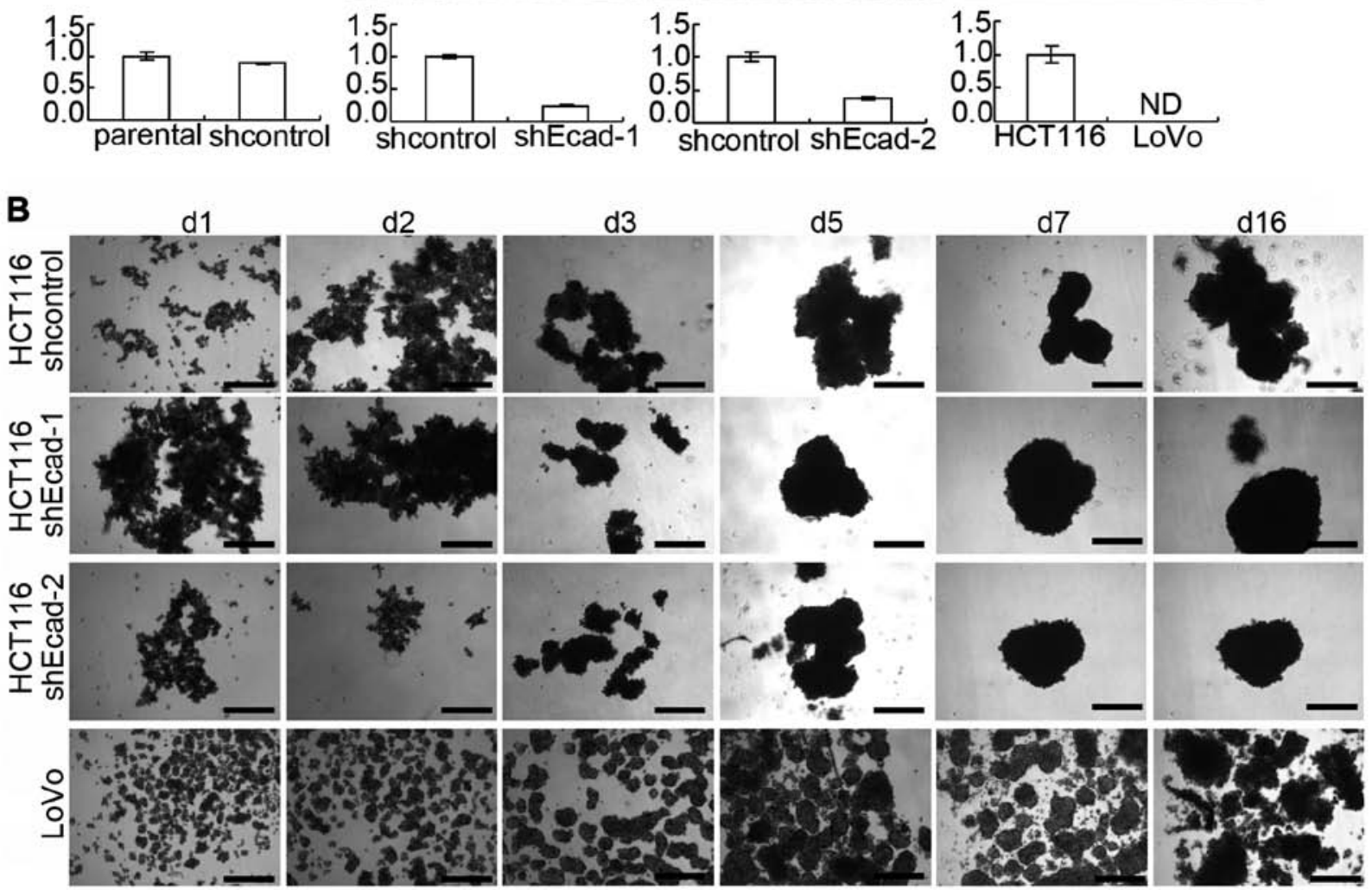

Figure 2. E-cadherin knockdown does not change the architecture formation of 3D cultures, as assessed under an invert microscope. (A) E-cadherin protein level was assayed by western blotting. The protein was extracted from $2 \mathrm{D}$ cultures grown for 3 days or from $3 \mathrm{D}$ cultures grown for 5 days. Relative quantities of E-cadherin of each group were normalized to $\beta$-actin and diagramed. ND, not detectable. Each group contained 3 independent samples. (B) shcontrol HCT116, HCT116 with E-cadherin knockdown (shEcad) and LoVo cells were cultured under 3D condition and were photographed at different time-points. Scale bars represent $400 \mu \mathrm{m}$ (d, day).

structures mimic tumors at avascular stage or avascular tumor regions (Fig. 1E). HCT116 3D cultures were also observed using a transmission electron microscope. Cell-cell junctions, including tight junctions, gap junctions, intermediate junctions and desmosomes, were commonly found in 3D cultures. Cellcell junctions were found in most of cells (Fig. 1F-H).

E-cadherin knockdown does not change the architecture formation of $3 D$ cultures. E-cadherin is expressed by a variety of tissues and plays a key role in mediating cell-cell adhesive systems $(9,10)$. It was reported that inhibition of E-cadherin function in cell-cell adhesion by E-cadherin neutralizing antibody (SHE78-7) disrupted preformed colorectal cancer cell 3D cultures $(6,13)$. In this study, E-cadherin in HCT116 was knocked down by lentiviral delivery of shRNA. The efficiency was confirmed by western blotting (Fig. 2A). HCT116 with E-cadherin knockdown (shEcad) grew as multicellular spheroids as HCT116 transfected with control lentivirus for 16 days and no difference was observed under an invert microscope (Fig. 2B). LoVo cells were also employed because E-cadherin expression in LoVo was too low to be detected using western blotting (Fig. 2A). LoVo cells also automatically formed spheroids under 3D culture conditions (Fig. 2B). The above suggested that E-cadherin knockdown does not change the architecture formation of 3D cultures, as assessed under the inverted microscope.

E-cadherin knockdown in HCT116 reduces chemosensitivity only in $3 D$ cultures. Intercellular force and homophilic binding of E-cadherin on adjacent cells trigger certain E-cadherin signaling cascades $(9,10,12)$. Therefore, the function of E-cadherin in 3D cultures may be different from in $2 \mathrm{D}$ cultures. Thus, the role of E-cadherin in chemosensitivity in $3 \mathrm{D}$ cultures and in $2 \mathrm{D}$ cultures was explored.

WST assay was performed to evaluate sensitivity to anticancer drugs (Fig. 3A). The viability of parental HCT116 3D cultures treated with $80 \mathrm{mg} / \mathrm{l} 5-\mathrm{FU}$ for $24 \mathrm{~h}$ was $45.7 \pm 3.1 \%$ and that of shcontrol was $47.9 \pm 3.3 \%$. There was no significant difference $(p \geq 0.05)$. Compare to the shcontrol, the viability of shEcad-1 HCT116 3D cultures treated with $80 \mathrm{mg} / \mathrm{l}$ 5-FU increased to $66.0 \pm 2.9 \%(\mathrm{p}<0.01)$ and that of shEcad-2 increased to $61.8 \pm 2.5 \%(\mathrm{p}<0.01)$. E-cadherin knockdown also decreased chemosensitivity to CPT-11. The viabilities of parental, shcontrol, shEcad-1 and shEcad-2 treated with 

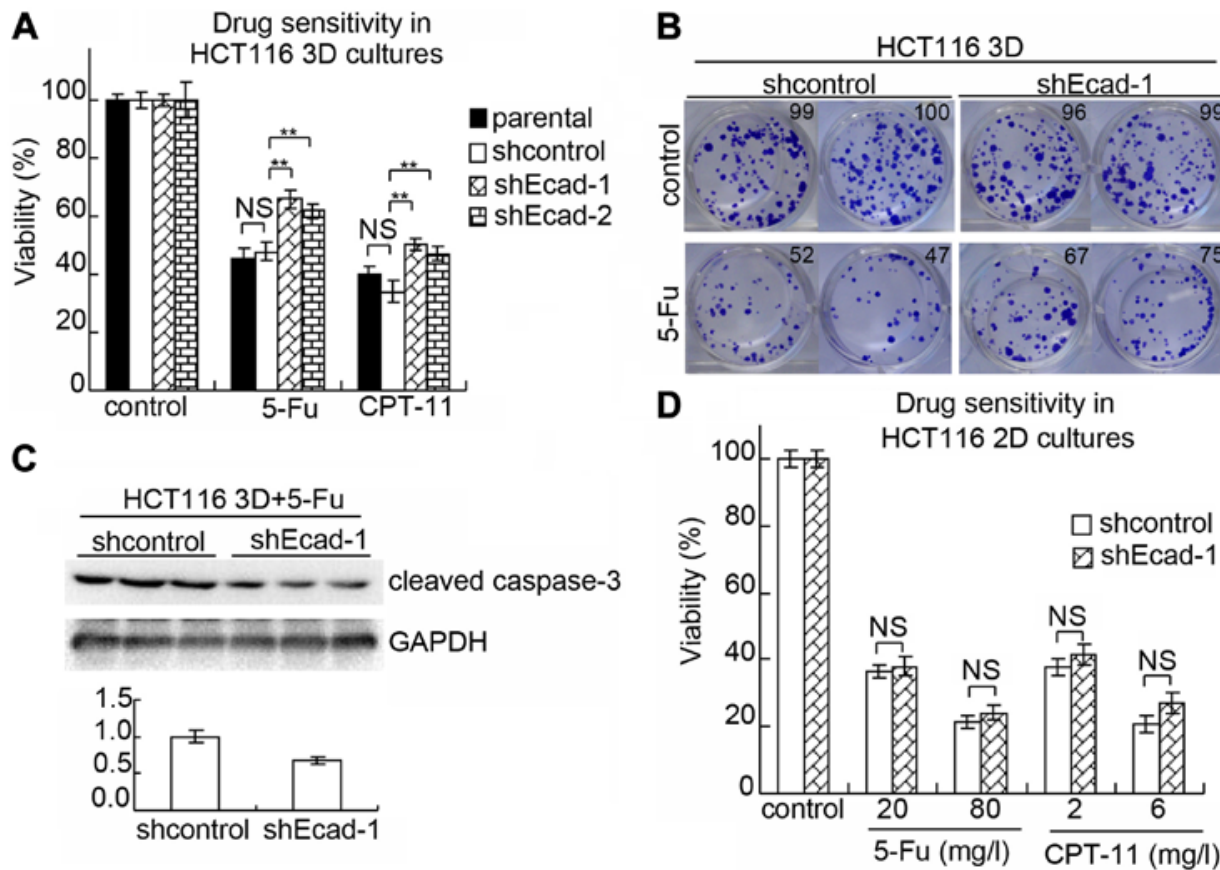

Figure 3. E-cadherin knockdown in HCT116 reduces chemosensitivity only in 3D cultures. (A) WST assay. E-cadherin knockdown reduced chemosensitivity in 3D cultures. ${ }^{* *} \mathrm{p}<0.01$. NS, not significant. (B) Clonogenic assay. The number of clones is marked on the top right of each image. E-cadherin knockdown increased the clonogenicity in 3D cultures. (C) Western blotting. Relative quantities of cleaved caspase-3 were normalized to GAPDH and diagramed. E-cadherin knockdown decreased 5-FU induced cleaved caspase-3. Each group contained 3 independent samples. (D) WST assay. E-cadherin knockdown did not significantly changed chemosensitivity in 2D cultures. NS, not significant.

A
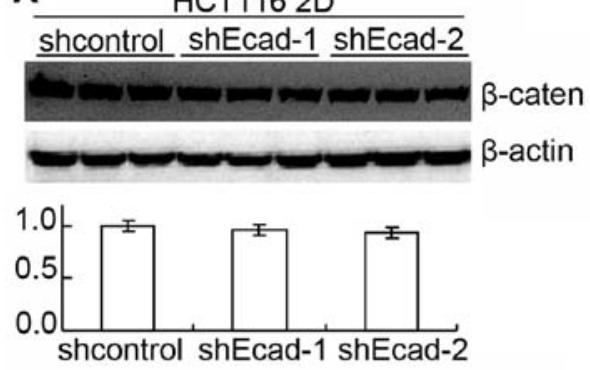

C
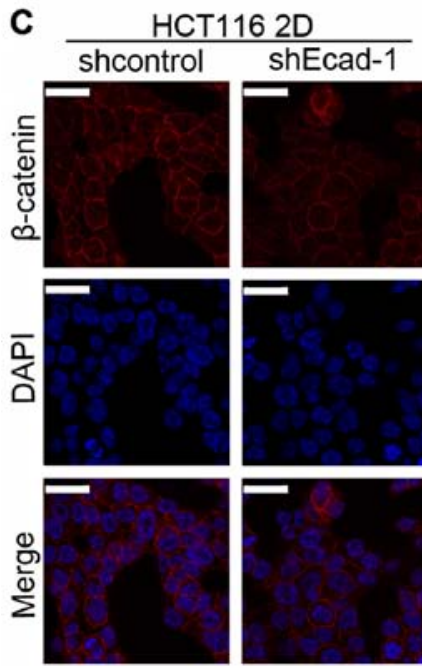

\section{D}

B
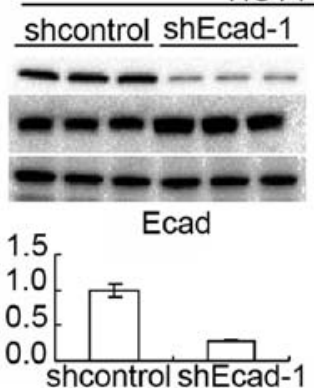

$\beta$-caten

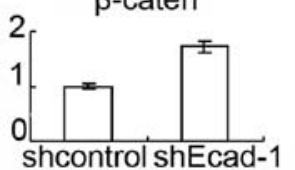

HCT116 3D
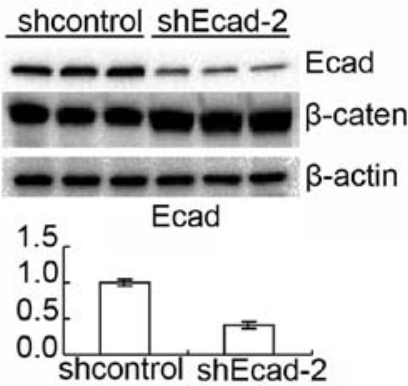

$\beta$-caten

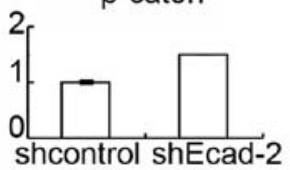

\section{HCT116 3D}

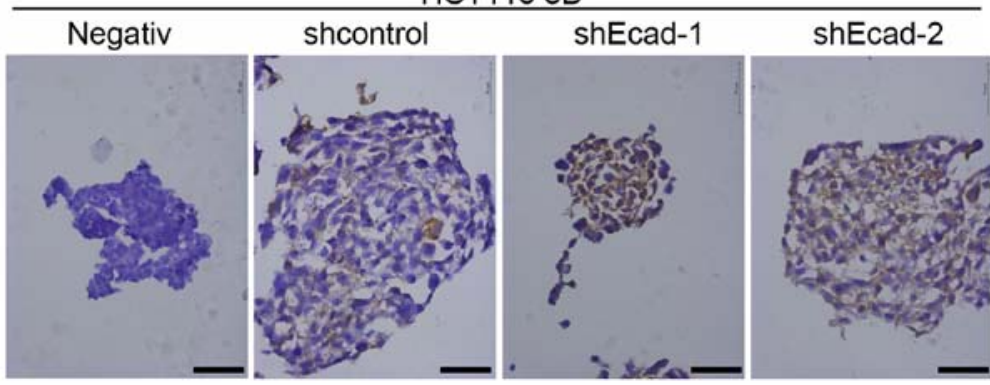

Figure 4. E-cadherin knockdown in HCT116 increases $\beta$-catenin only in 3D cultures. (A) Western blotting. Relative quantities of E-cadherin were normalized to $\beta$-actin and diagramed. E-cadherin knockdown in HCT116 2D cultures did not detectably change $\beta$-catenin expression. Each group contained 3 independent samples. (B) Western blotting. Relative quantities of bands were normalized to $\beta$-actin and diagramed. E-cadherin knockdown in HCT116 3D cultures increased $\beta$-catenin. Each group contained 3 independent samples. (C) Immunofluorescence of $\beta$-catenin in HCT116 2D cultures. E-cadherin knockdown in HCT116 2D cultures did not detectably change $\beta$-catenin expression. Scale bars represent $25 \mu \mathrm{m}$. (D) Immunohistochemistry of $\beta$-catenin in HCT116 3D cultures. E-cadherin knockdown in HCT116 3D cultures increased $\beta$-catenin. Scale bars represent $50 \mu \mathrm{m}$. 
A
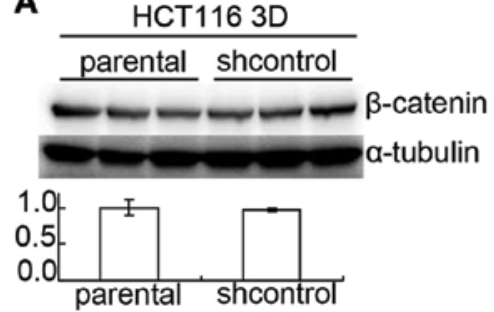

LoVo 3D
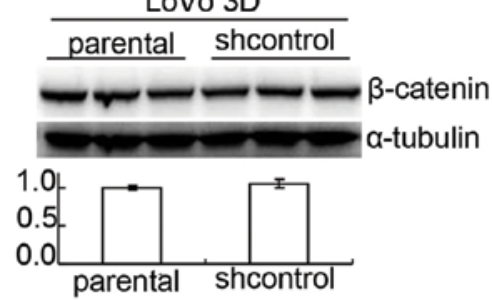

HCT116 3D

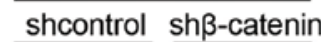

B

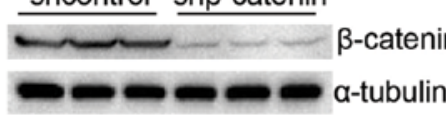

1.0 .

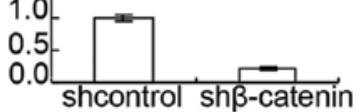

LoVo 3D
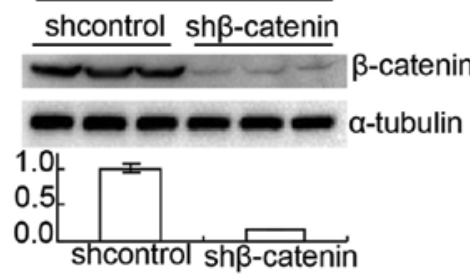

Drug sensitivity in 3D

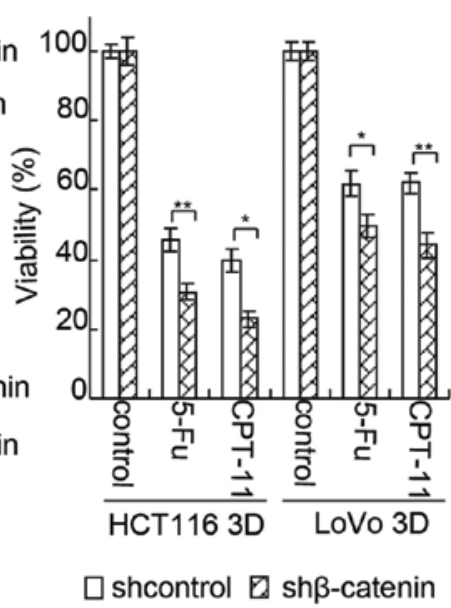

C

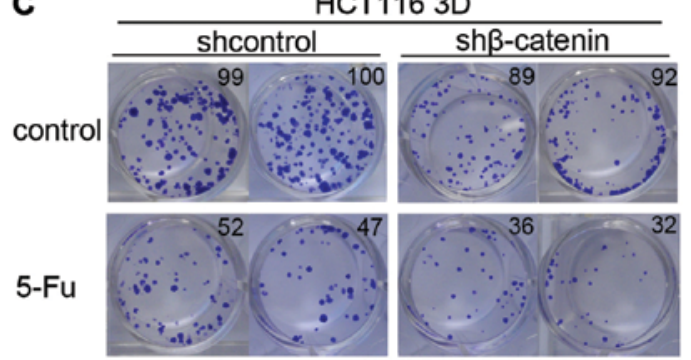

D

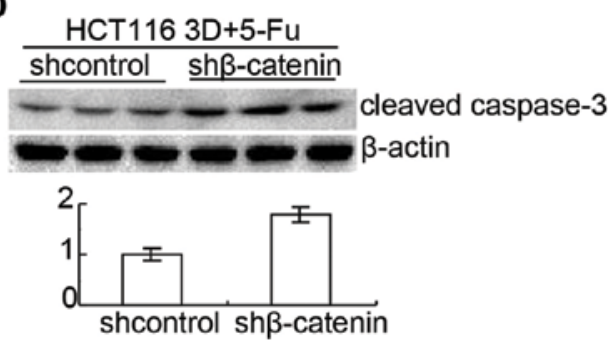

Figure 5. Knockdown of $\beta$-catenin increases chemosensitivity in 3D cultures. (A) Efficiency of $\beta$-catenin knockdown by shRNA in HCT116 and LoVo was assayed by western blotting. Relative quantities of $\beta$-catenin were normalized to $\alpha$-tubulin and diagramed. Each group contained 3 independent samples. (B) WST assay. Knockdown of $\beta$-catenin enhanced chemosensitivity in $3 \mathrm{D}$ cultures. ${ }^{*} \mathrm{p}<0.05,{ }^{* *} \mathrm{p}<0.01$. (C) Clonogenic assay. The number of clones is marked on the top right of each image. Knockdown of $\beta$-catenin enhanced chemosensitivity in HCT116 3D cultures. (D) Cleaved caspase-3 was assayed by western blotting. Relative quantities of cleaved caspase-3 were normalized to $\beta$-actin and diagramed. Knockdown of $\beta$-catenin increased 5 -FU induced cleaved caspase-3. Each group contained 3 independent samples.

$6 \mathrm{mg} / \mathrm{l} \mathrm{CPT}-11$ were $39.7 \pm 3.1,34.0 \pm 3.7$ (vs parental: $\mathrm{p} \geq 0.05$ ), $50.6 \pm 2.0$ (vs shcontrol: $\mathrm{p}<0.01$ ) and $47.1 \pm 2.3 \%$ (vs shcontrol: $\mathrm{p}<0.01)$.

Results from the clonogenic assay were consistent with the WST assay.E-cadherin knockdown increased the clonogenicity (Fig. 3B). Cleaved caspase-3 of shcontrol HCT116 3D cultures and shEcad-1 HCT116 3D cultures treated with $20 \mathrm{mg} / \mathrm{l} 5-\mathrm{FU}$ for $24 \mathrm{~h}$ was analyzed by western blotting. Result showed that E-cadherin knockdown decreased cleaved caspase-3 (Fig. 3C).

To explore the effect of E-cadherin knockdown on chemosensitivity in 2D cultures, sensitivity was performed by WST assay (Fig. 3D). The viability of parental, shcontrol and shEcad-1 HCT116 cells, respectively, treated with $20 \mathrm{mg} / 1$ 5-FU was $35.0 \pm 2.3,36.4 \pm 2.0$ (vs parental: $\mathrm{p} \geq 0.05$ ) and $37.9 \pm 2.9 \%$ (vs shcontrol: $\mathrm{p} \geq 0.05$ ). The viability of parental, shcontrol and shEcad-1 treated with $80 \mathrm{mg} / 15-\mathrm{FU}$ was $23.3 \pm 2.1,21.3 \pm 2.1$ (vs parental: $\mathrm{p} \geq 0.05$ ) and $24.2 \pm 2.4 \%$ (vs shcontrol: $\mathrm{p} \geq 0.05$ ). The viability, respectively, of parental, shcontrol and shEcad-1 treated with $2 \mathrm{mg} / \mathrm{l}$ CPT-11 was $40.0 \pm 1.8,37.9 \pm 2.5$ (vs parental: $\mathrm{p} \geq 0.05$ ) and $41.5 \pm 3.2 \%$ (vs shcontrol: $\mathrm{p} \geq 0.05$ ). The viabilities of parental, shcontrol and shEcad-1 treated with $6 \mathrm{mg} / \mathrm{l} \mathrm{CPT-11}$ were $24.5 \pm 2.7$, $21.0 \pm 2.5$ (vs parental: $\mathrm{p} \geq 0.05$ ) and $27.2 \pm 3.1 \%$ (vs shcontrol: $\mathrm{p} \geq 0.05$ ). Thus, E-cadherin knockdown does not significantly change chemosensitivity in HCT116 2D cultures.
E-cadherin knockdown increases $\beta$-catenin reducing chemosensitivity only in $3 D$ cultures. Protein $\beta$-catenin was reported to interact with E-cadherin and was also involved in cell adhesion $(10,22)$. Western blotting showed that E-cadherin knockdown increased $\beta$-catenin in 3D cultures but did not detectably increased $\beta$-catenin in $2 \mathrm{D}$ cultures (Fig. $4 \mathrm{~A}$ and $\mathrm{B}$ ). To confirm the result, $\beta$-catenin was assayed by immunofluorescence in 2D cultures and immunohistochemistry in $3 \mathrm{D}$ cultures. Immunofluorescence showed that $\beta$-catenin was not significantly changed by E-cadherin knockdown in 2D cultures (Fig. 4C) while immunohistochemistry showed that E-cadherin knockdown increased $\beta$-catenin in 3D cultures (Fig. 4D).

As mentioned above, E-cadherin knockdown increased $\beta$-catenin and decreased chemosensitivity only in 3D cultures. Since it was reported that enhancing $\beta$-catenin expression promoted anticancer drug resistance (23), $\beta$-catenin may be involved in the mechanism of E-cadherin knockdown decreasing chemosensitivity in 3D cultures. To confirm this, $\beta$-catenin in HCT116 and LoVo was knocked down by lentiviral delivery of shRNA, respectively. After the efficiency was confirmed by western blotting (Fig. 5A), chemoensitivity to 5-FU and CPT-11 in 3D cultures was evaluated by WST assay (Fig. 5B). The viability of shcontrol HCT116 treated with $80 \mathrm{mg} / \mathrm{l} 5$-FU was $45.7 \pm 3.1 \%$ while that of $\beta$-catenin knock- 
A

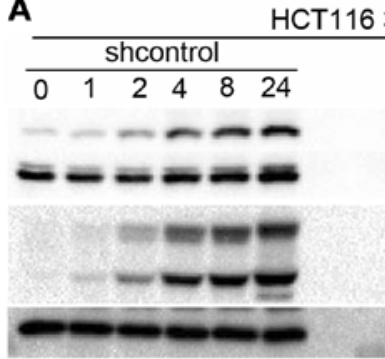

p-p38
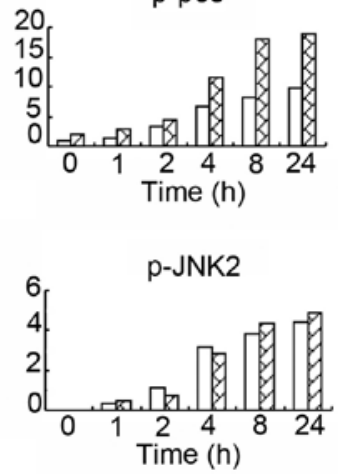

$\square$ shcontrol घ shEcad-1 c

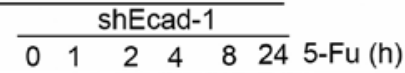

- - - - p-p38

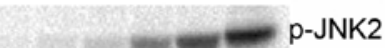

p-JNK1

GAPDH
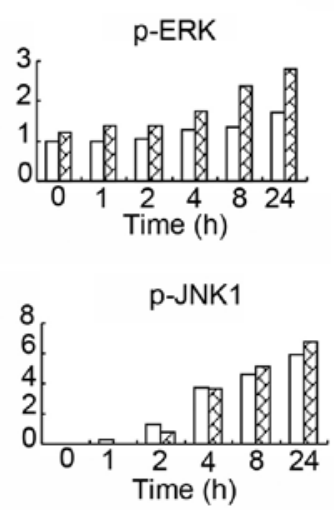

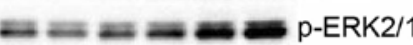

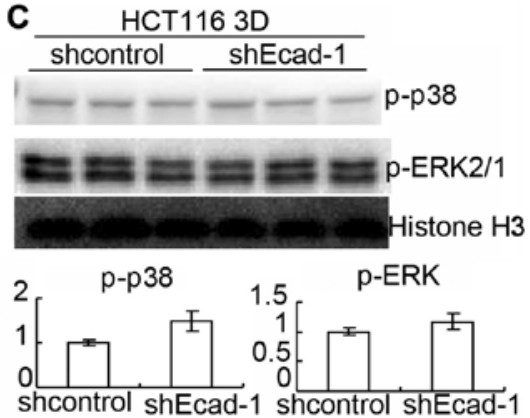

D
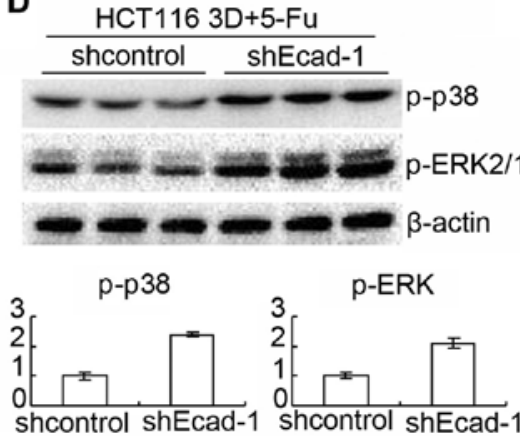

$\mathbf{F}$

HCT116 2D+5-Fu shcontrol shEcad-1

B

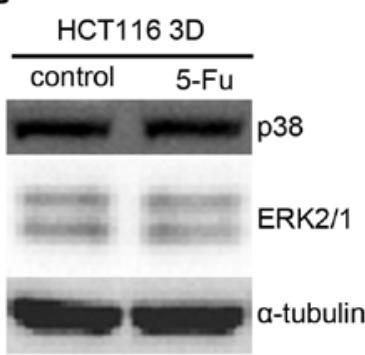

E

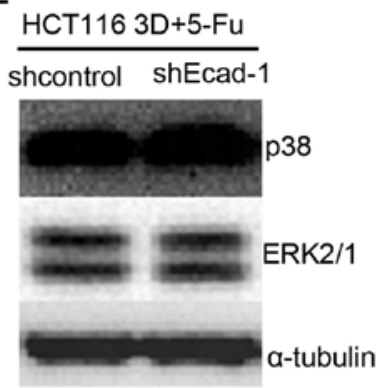

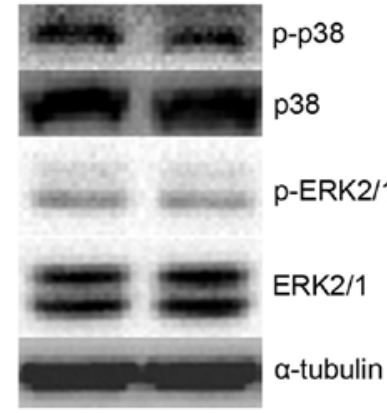

Figure 6. E-cadherin knockdown increased p38 and ERK1/2 activation in HCT116 3D cultures. (A) Western blotting. Relative quantities of bands were normalized to GAPDH and diagramed. The protein levels of p-p38, p-ERK2/1 and p-JNK2/1 were increased by 5-FU treatment in a time-dependent manner. E-cadherin knockdown increased p-p38, p-ERK2/1 except p-JNK2/1. (B) Western blotting. Total p38 and ERK1/2 were not detectably changed by 5-FU treatment. (C) Basal p-p38 and p-ERK1/2 were assayed by western blotting. Relative quantities of bands were normalized to histone H3 and diagramed. Each group contained 3 independent samples. (D) Western blotting. Relative quantities of bands were normalized to $\beta$-actin and diagramed. Each group contained 3 independent samples. (E) Western blotting. Total p38 and ERK1/2 were not detectably changed by E-cadherin knockdown in 3D cultures. (F) Western blotting. E-cadherin knockdown did not detectably change p-p38, p38, p-ERK1/2 and ERK1/2 in 2D cultures.

down $(\operatorname{sh} \beta$-catenin) HCT116 was $30.8 \pm 2.1 \%(\mathrm{p}<0.01)$. The viability of shcontrol HCT116 treated with $6 \mathrm{mg} / \mathrm{l} \mathrm{CPT}-11$ was $39.7 \pm 3.1 \%$ while that of $\operatorname{sh} \beta$-catenin was $22.9 \pm 2.2 \%(\mathrm{p}<0.05)$. Results in LoVo 3D cultures were similar to those in HCT116. The viability of shcontrol LoVo treated with $80 \mathrm{mg} / \mathrm{l} 5-\mathrm{FU}$ was $61.9 \pm 3.4 \%$ while that of $\operatorname{sh} \beta$-catenin was $49.6 \pm 3.3 \%(\mathrm{p}<0.05)$. The viability of shcontrol LoVo treated with $6 \mathrm{mg} / \mathrm{l}$ CPT-11 was $62.1 \pm 3.0 \%$ while that of $\operatorname{sh} \beta$-catenin was $44.2 \pm 3.7 \%$ $(\mathrm{p}<0.01)$.

Results from clonogenic assay were consistent with WST assay. Knockdown of $\beta$-catenin decreased the clonogenicity (Fig. 5C). It seemed that knockdown of $\beta$-catenin decreased both the size of clones and the clonogenicity without 5-FU treatment. The ratio of clonogenicity with 5-FU:clonogenicity without 5 -FU was lower in the $\operatorname{sh} \beta$-catenin group than in the shcontrol group. Cleaved caspase-3 of shcontrol HCT116 3D cultures and $\operatorname{sh} \beta$-catenin HCT116 3D cultures treated with $20 \mathrm{mg} / 15-\mathrm{FU}$ for $24 \mathrm{~h}$ was analyzed by western blotting. Result showed that knockdown of $\beta$-catenin increased cleaved caspase-3 (Fig. 5D).

The above suggests that E-cadherin knockdown increases $\beta$-catenin to reduce chemosensitivity in $3 \mathrm{D}$ cultures.

MAPK pathway is involved in mechanism of E-cadherin knockdown increasing $\beta$-catenin to reduce chemosensitivity. MAPK signaling plays a critical role in the sensitivity to anticancer therapies (4,24-28). Also, formation of E-cadherinmediated cell-cell adhesion regulates MAPKs (29). Thus, the role of MAPKs in E-cadherin knockdown increasing $\beta$-catenin to reduce chemosensitivity was explored. HCT116 3D cultures were treated with $80 \mathrm{mg} / 1$ 5-FU for different time points. Protein p-p38, p-extracellular-signal-regulated kinase (ERK) 
A

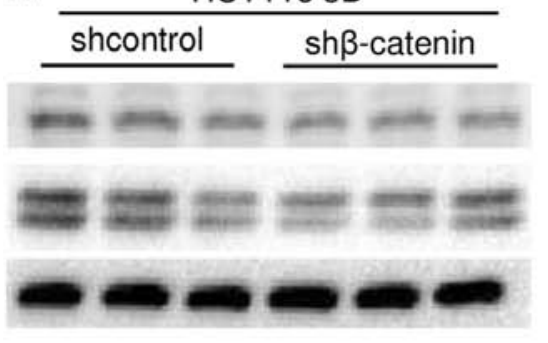
HCT116 3D

\section{B}

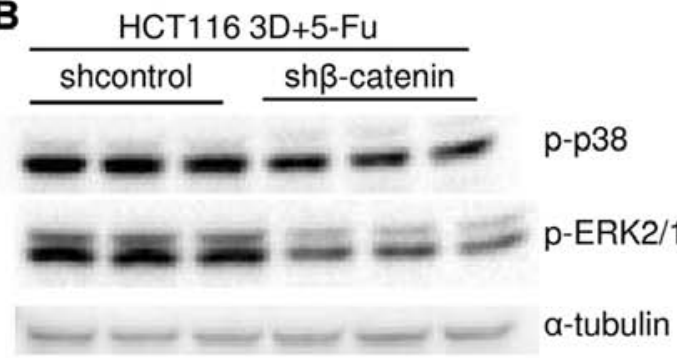

p-p38
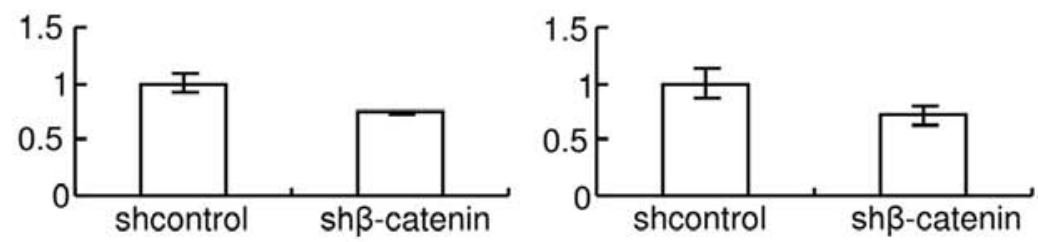

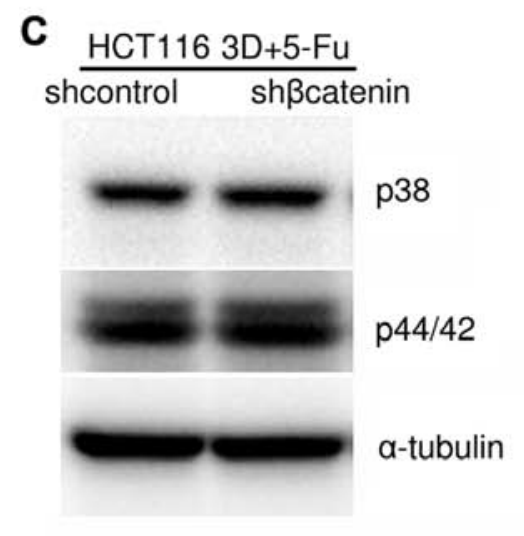

D
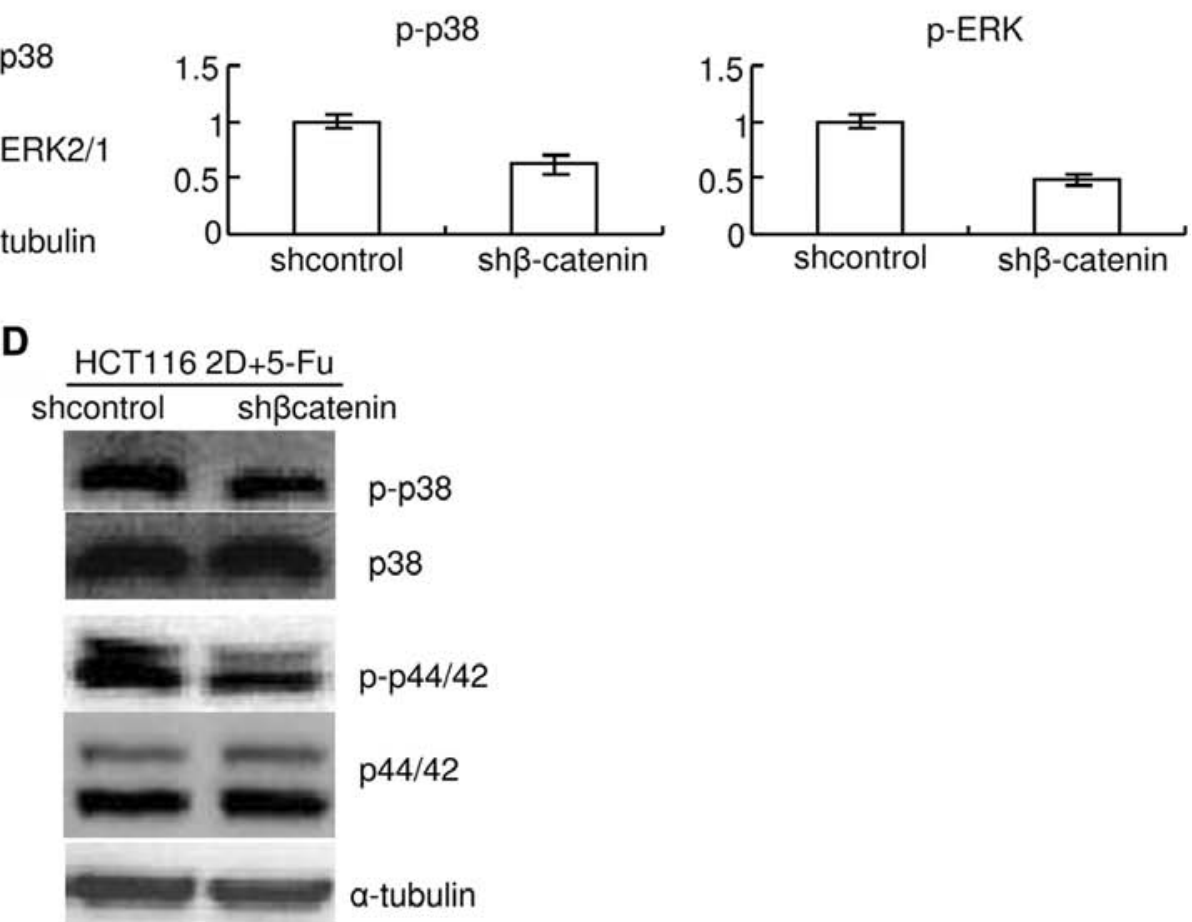

Figure 7. Knockdown of $\beta$-catenin decreases p-p38 and p-ERK1/2. (A and B) Western blotting. Each group contained 3 independent samples. Basal p-p38 and p-ERK1/2 (A) or p-p38 and p-ERK1/2 induced by 5-FU (B) in 3D cultures were assayed. Relative quantities of bands were normalized to $\alpha$-tubulin and diagramed. (C) Western blotting. Knockdown of $\beta$-catenin did not detectably change p38 or ERK1/2 induced by 5-FU in 3D cultures. (D) Western blotting. Knockdown of $\beta$-catenin decreased p-p38 and p-ERK1/2 induced by 5-FU in 2D cultures and did not detectably change p38 or ERK1/2.

$1 / 2$ and p-c-Jun N-terminal kinase (JNK) $1 / 2$ were assayed by western blotting. 5-FU treatment increased p-p38, p-ERK $1 / 2$ and p-JNK $1 / 2$ in a time dependent manner in HCT116 3D cultures (Fig. 6A). E-cadherin knockdown increased p-p38 and p-ERK 1/2, except JNK1/2 (Fig. 6A). Total p38 and ERK1/2 protein level was not detectably changed by $80 \mathrm{mg} / 1$ 5-FU treatment for $8 \mathrm{~h}$ (Fig. 6B). E-cadherin knockdown mildly increased basal levels of p-p38 and p-ERK1/2 (Fig. 6A and C). When treated with $80 \mathrm{mg} / \mathrm{l} 5$-FU for $8 \mathrm{~h}$, E-cadherin knockdown increased p-p38 and p-ERK1/2 in HCT116 3D cultures (Fig. 6A and D). E-cadherin knockdown neither changed total p38 and ERK1/2 protein level in 2D cultures nor in 3D cultures under treatment of 5-FU (Fig. 6E and F). E-cadherin knockdown did not detectably change p-p38 and p-ERK1/2 protein level in 2D cultures, either (Fig. 6F). Knockdown of $\beta$-catenin also mildly decreased basal levels of p-p38 and p-ERK1/2 (Fig. 7A). Knockdown of $\beta$-catenin decreased p-p38 and p-ERK1/2 induced by $80 \mathrm{mg} / \mathrm{l} 5$-FU 8 -h treatment both in $3 \mathrm{D}$ cultures and in $2 \mathrm{D}$ cultures (Fig. 7B and D). Total p38 or ERK1/2 protein level was not detectably changed by $\beta$-catenin knockdown (Fig. 7C and D). The results together with the above indicate that E-cadherin knockdown increases $\beta$-catenin only in $3 \mathrm{D}$ cultures but $\beta$-catenin enhances p-p38 and p-ERK1/2 both in 3D cultures and in 2D cultures.

SB202190 (p38 inhibitor) (30) and U0126 (ERK1/2 inhibitor) (31) were used to inhibit activations of p38 and ERK1/2 in HCT116 3D cultures, respectively. HCT116 3D cultures were treated with $20 \mu \mathrm{M}$ SB202190, $20 \mu \mathrm{M}$ U0126 or DMSO $1 \mathrm{~h}$ following treatment of $80 \mathrm{mg} / 15$-FU for different time periods, respectively. Western blotting showed that SB202190 remarkably inhibited p-p38 and U0126 remarkably inhibited p-ERK1/2 (Fig. 8A and B). WST showed that inhibition of $\mathrm{p} 38$ or ERK $1 / 2$ activation significantly increased HCT116 3D cultures chemosensitivity to 5-FU, respectively (Fig. 8C). HCT116 3D cultures were treated with $20 \mu \mathrm{M}$ SB202190, $20 \mu \mathrm{M}$ U0126 or DMSO $1 \mathrm{~h}$ following treatment of $80 \mathrm{mg} / 1$ 5-FU for $24 \mathrm{~h}$, respectively. The viabilities of DMSO, SB202190 and U0126 were 44.1 $\pm 3.5,26.6 \pm 2.3$ (vs DMSO: 
A

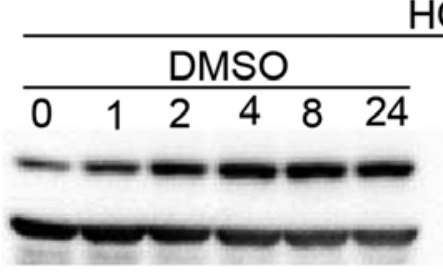

HCT116 3D

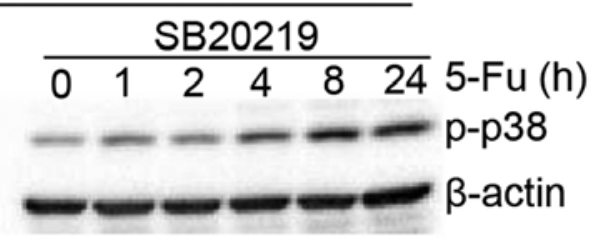

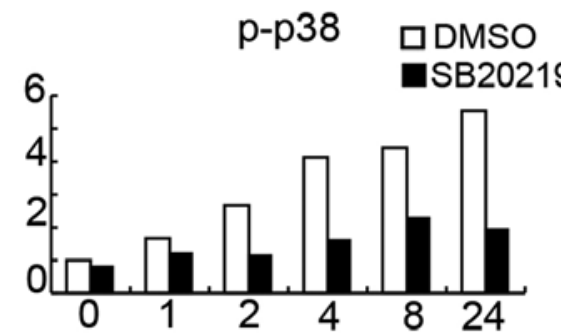

C

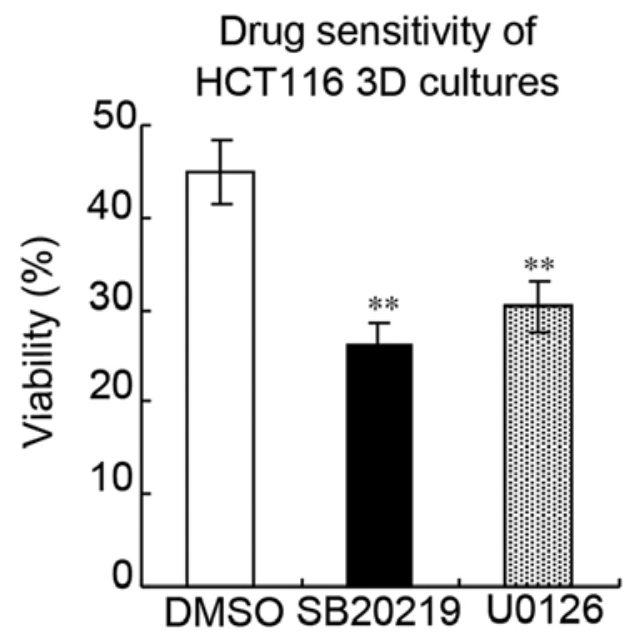

B

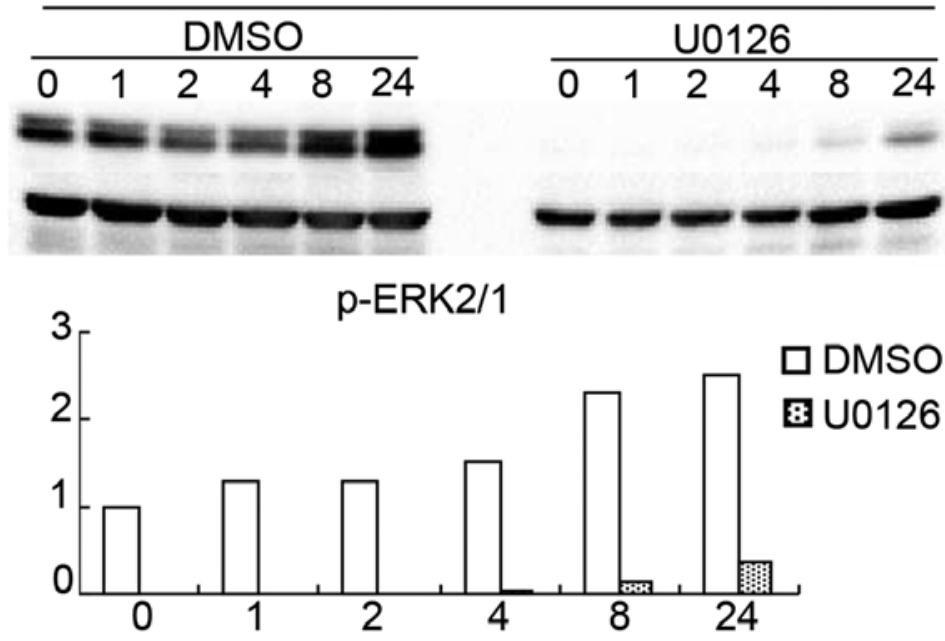

$5-\mathrm{Fu}(\mathrm{h})$ p-ERK2/1

Figure 8. Inhibition of p38 or ERK1/2 activation enhances drug sensitivity. (A) Protein p-p38 was assayed by western blotting. Relative quantities of p-p38 were normalized to $\beta$-actin and diagramed. (B) Protein p-ERK1/2 was assayed by western blotting. Relative quantities of p-ERK1/2 were normalized to $\beta$-actin and diagramed. (C) WST assay. Inhibition of p38 or ERK1/2 activation enhanced drug sensitivity. ${ }^{* *} \mathrm{p}<0.01$.

$\mathrm{p}<0.01$ ), and $30.0 \pm 2.6 \%$ (vs DMSO: $\mathrm{p}<0.01$ ), thus suggesting that MAPK pathway is involved in E-cadherin knockdown increasing $\beta$-catenin to reduce chemosensitivity.

\section{Discussion}

Colorectal cancer is one of the most lethal diseases of all malignancies world-wide $(32,33)$. The incidence of colorectal cancer in developing countries is increasing, partly attributing to lipid metabolism $(19,32,33)$. More than $35 \%$ of the patients die within 5 years after diagnosis even in developed countries (32,33). This discouraging fact is largely due to the ability of a malignant tumor to demonstrate resistance to chemotherapies new, and old $(4,5,25)$. Accumulating evidence indicates that microenvironment influences tumor cells biological behavior, including chemosensitivity $(4,5)$. Among the myriad of microenvironmental factors impacting on cancer cell chemosensitivity, cell-cell adhesion has recently been identified as key determinant (1-2,4-6,25). In 3D cultures, cells adhered to each other within layers of cells. All types of cell-cell junctions, including tight junctions, gap junctions, intermediate junctions and desmosomes, were commonly found (Fig. 1). Their structures are very similar to tumors at avascular stage or avascular tumor regions (Fig. 1) $(1,3,10,17)$, indicating 3D cultures have the potential to bridge the gap between monolayer cultures and xenografts for deciphering the function of cell-cell adhesive systems $(1,3,10,17)$.

E-cadherin plays a key role in mediating cell-cell adhesive systems $(2,6,9,10)$. It was reported that inhibition of E-cadherin function in cell-cell adhesion only by E-cadherin neutralizing antibody (SHE78-7) disrupted preformed 3D cultures $(6,13)$. However, E-cadherin knockdown did not prevent suspension of HCT116 from 3D culture formation. E-cadherin protein in LoVo cells was too low to be detected using western blotting while LoVo cells formed 3D cultures (Fig. 2). It was also reported that prostate cancer cell line PC-3 with E-cadherin epigenetically silenced, could form 3D cultures $(13,34)$. Therefore, E-cadherin may not be essential for architecture formation of $3 \mathrm{D}$ cultures in vitro as tumors in vivo.

Decreased E-cadherin expression correlates with poor prognosis in patients with colorectal cancer (15). Intercellular force and homophilic binding of cadherin on adjacent cells trigger E-cadherin to interact with certain proteins, for example $\beta$-catenin, to activate signaling cascades $(9,10,12,22)$. Since 3D cultures better reflect cell-cell adhesion and chemosensitivity in vivo tumors (Fig. 1) $(1,2,4-8,13,17)$ the role of E-cadherin in colorectal cancer chemosensitivity was explored in 3D cultures. E-cadherin knockdown significantly decreased chemosensitivity to anticancer drugs in 3D cultures but did not significantly change chemosensitivity in 2D cultures (Fig. 3).

Under chemotherapy, the molecular mechanisms deciding whether a tumor cell commits to cell death or survives are complex. $\beta$-catenin plays a critical role in survival and is involved in adhesion system $(10,22,23)$ E-cadherin cytoplasmic domain contains a catenin-binding domain $(10,22)$. E-cadherin knockdown increased $\beta$-catenin in 3D cultures and $\beta$-catenin knockdown significantly enhanced chemosensitivity (Figs. 3-5). In 2D cultures, E-cadherin knockdown did not 
detectably change $\beta$-catenin (Fig. 4), suggesting E-cadherin knockdown increases $\beta$-catenin to decrease chemosensitivity only in 3D cultures.

Mounting evidence indicates a critical role of apoptotic pathways in determining the response of human cancers to anticancer drugs, including 5-FU $(3,25,35,36)$. Caspase- 3 plays a key role in apoptosis induced by $5-\mathrm{FU}$ and cleaved caspase-3 is considered as an apoptotic marker (35). E-cadherin knockdown significantly decreased cleaved caspase-3 induced by 5 -FU and $\beta$-catenin knockdown significantly increased cleaved caspase-3 (Figs. 3C and 5D). Thus, suggesting apoptosis is involved in the mechanism of E-cadherin knockdown increasing $\beta$-catenin to reduce chemosensitivity.

Among the many cell signaling transduction pathways regulating apoptosis, the MAPK signaling pathway plays an important role in the sensitivity to anticancer therapies $(27,28,30,31,37)$ Also, formation of E-cadherin-mediated cell-cell adhesion regulates MAPKs (29). However, the role of MAPKs in cancer is as pleiotropic as cancer itself $(27-31,38)$. In this study, p38, ERK1/2 and JNK1/2 were dramatically activated by chemotherapy (Fig. 6A and B). E-cadherin knockdown enhanced chemotherapy-induced p-p38 and p-ERK1/2, except p-JNK1/2 only in 3D cultures (Fig. 6). Knockdown of $\beta$-catenin attenuated chemotherapy-induced p-p38 and p-ERK1/2 both in 3D cultures and in 2D cultures (Fig. 7). Treatment of 3D cultures with SB202190 to inhibit p38 activation or U0126 to inhibit ERK1/2 activation significantly increased chemosensitivity, respectively (Fig. 7). The above suggests E-cadherin knockdown increases $\beta$-catenin to reduce chemosensitivity only in $3 \mathrm{D}$ cultures and $\beta$-catenin increasing p-p38/p-ERK1/2 is involved in the mechanism though the $\beta$-catenin-MAPK pathway is not unique in $3 \mathrm{D}$ cultures.

In conclusion, 3D cultures consist of layers of cells, preserving cell-cell adhesive systems. These allow a good model to decipher the function of cell-cell adhesive systems in cancer. Intercellular adhesion triggers certain E-cadherin signaling cascades $(9,10,12)$. Data in this study indicate that E-cadherin knockdown significantly increases $\beta$-catenin resulting in decrease of chemosensitivity in 3D cultures but this effect was not detected in $2 \mathrm{D}$ cultures. $\beta$-catenin enhancing the p-p38/p-ERK1/2 is involved in this mechanism, though the $\beta$-catenin-MAPK pathway is not unique in 3D cultures.

\section{Acknowledgements}

This study was supported by the National Natural Science Foundation of China (grant no. 81000990), the Natural Science Foundation Project of CQ CSTC (grant no. 2009BB5339) and the Science Foundation of Third Military Medical University for the Young Scholar (grant no. 2009XQN32).

\section{References}

1. Shamir ER and Ewald AJ: Three-dimensional organotypic culture: Experimental models of mammalian biology and disease. Nat Rev Mol Cell Biol 15: 647-664, 2014.

2. Kang HG, Jenabi JM, Zhang J, Keshelava N, Shimada H, May WA, Ng T, Reynolds CP, Triche TJ and Sorensen PH: E-cadherin cell-cell adhesion in ewing tumor cells mediates suppression of anoikis through activation of the ErbB4 tyrosine kinase. Cancer Res 67: 3094-3105, 2007.
3. Desoize B and Jardillier J: Multicellular resistance: A paradigm for clinical resistance? Crit Rev Oncol Hematol 36: 193-207, 2000.

4. He JM, Wang FC, Qi HB, Li Y and Liang HJ: Down-regulation of alphav integrin by retroviral delivery of small interfering RNA reduces multicellular resistance of HT29. Cancer Lett 284: 182-188, 2009.

5. Kobayashi H, Man S, Graham CH, Kapitain SJ, Teicher BA and Kerbel RS: Acquired multicellular-mediated resistance to alkylating agents in cancer. Proc Natl Acad Sci USA 90: 3294-3298, 1993.

6. Green SK, Karlsson MC, Ravetch JV and Kerbel RS: Disruption of cell-cell adhesion enhances antibody-dependent cellular cytotoxicity: Implications for antibody-based therapeutics of cancer. Cancer Res 62: 6891-6900, 2002.

7. Yang Z and Zhao X: A 3D model of ovarian cancer cell lines on peptide nanofiber scaffold to explore the cell-scaffold interaction and chemotherapeutic resistance of anticancer drugs. Int J Nanomedicine 6: 303-310, 2011.

8. Lee JM, Mhawech-Fauceglia P, Lee N, Parsanian LC, Lin YG, Gayther SA and Lawrenson K: A three-dimensional microenvironment alters protein expression and chemosensitivity of epithelial ovarian cancer cells in vitro. Lab Invest 93: 528-542, 2013.

9. Engl W, Arasi B, Yap LL, Thiery JP and Viasnoff V: Actin dynamics modulate mechanosensitive immobilization of E-cadherin at adherens junctions. Nat Cell Biol 16: 587-594, 2014.

10. Gall TM and Frampton AE: Gene of the month: E-cadherin (CDH1). J Clin Pathol 66: 928-932, 2013.

11. Mattias L, Haque A, Adnan N and Akaike T: The effects of artificial E-cadherin matrix-induced embryonic stem cell scattering on paxillin and RhoA activation via $\alpha$-catenin. Biomaterials 35: 1797-1806, 2014

12. Truffi M, Dubreuil V, Liang X, Vacaresse N, Nigon F, Han SP, Yap AS, Gomez GA and Sap J: RPTP $\alpha$ controls epithelial adherens junctions, linking E-cadherin engagement to c-Srcmediated phosphorylation of cortactin. J Cell Sci 127: 2420-2432, 2014.

13. Nakamura T, Kato Y, Fuji H, Horiuchi T, Chiba Y and Tanaka K: E-cadherin-dependent intercellular adhesion enhances chemoresistance. Int J Mol Med 12: 693-700, 2003.

14. Govatati S, Singamsetty GK, Nallabelli N, et al: Contribution of cyclin D1 (CCND1) and E-cadherin (CDH1) alterations to colorectal cancer susceptibility: A case-control study. Tumour Biol 35: 12059-12067, 2014.

15. Yun JA, Kim SH, Hong HK, Yun SH, Kim HC, Chun HK, Cho YB and Lee WY: Loss of E-Cadherin expression is associated with a poor prognosis in stage III colorectal cancer. Oncology 86: 318-328, 2014.

16. Li Y, He J, Zhong D, Li J and Liang H: High-mobility group box 1 protein activating nuclear factor- $\kappa \mathrm{B}$ to upregulate vascular endothelial growth factor $\mathrm{C}$ is involved in lymphangiogenesis and lymphatic node metastasis in colon cancer. J Int Med Res 43: 494-505, 2015.

17. Phung YT, Barbone D, Broaddus VC and Ho M: Rapid generation of in vitro multicellular spheroids for the study of monoclonal antibody therapy. J Cancer 2: 507-514, 2011.

18. Onder TT, Gupta PB, Mani SA, Yang J, Lander ES and Weinberg RA: Loss of E-cadherin promotes metastasis via multiple downstream transcriptional pathways. Cancer Res 68: 3645-3654, 2008.

19. He J, Shin H, Wei X, Kadegowda AK, Chen R and Xie SK: NPC1L1 knockout protects against colitis-associated tumorigenesis in mice. BMC Cancer 15: 189, 2015.

20. Franken NA, Rodermond HM, Stap J, Haveman J and van Bree C: Clonogenic assay of cells in vitro. Nat Protoc 1: 2315-2319, 2006.

21. Tanaka E, Hashimoto Y, Ito T, Kondo K, Higashiyama M, Tsunoda S, Ortiz C, Sakai Y, Inazawa J and Shimada Y: The suppression of aurora-A/STK15/BTAK expression enhances chemosensitivity to docetaxel in human esophageal squamous cell carcinoma. Clin Cancer Res 13: 1331-1340, 2007.

22. Lee DJ, Kang DH, Choi M, et al: Peroxiredoxin-2 represses melanoma metastasis by increasing E-cadherin/ $\beta$-catenin complexes in adherens junctions. Cancer Res 73: 4744-4757, 2013.

23. Liu Y, Ye X, Zhang JB, et al: PROX1 promotes hepatocellular carcinoma proliferation and sorafenib resistance by enhancing $\beta$-catenin expression and nuclear translocation. Oncogene: Feb 16, 2015 (Epub ahead of print) doi: 10.1038/onc.2015.7. 
24. Koti M, Gooding RJ, Nuin P, Haslehurst A, Crane C, Weberpals J, Childs T, Bryson P, Dharsee M, Evans K, et al: Identification of the IGF1/PI3K/NF $\kappa \mathrm{B} / \mathrm{ERK}$ gene signalling networks associated with chemotherapy resistance and treatment response in highgrade serous epithelial ovarian cancer. BMC Cancer 13: 549, 2013.

25. Liu S, Wang J, Niu W, Liu E, Wang J, Peng C, Lin P, Wang B, Khan AQ, Gao $\mathrm{H}$, et al: The $\beta 6$-integrin-ERK/MAP kinase pathway contributes to chemo resistance in colon cancer. Cancer Lett 328: 325-334, 2013.

26. Basile KJ, Abel EV, Dadpey N, Hartsough EJ, Fortina P and Aplin AE: In vivo MAPK reporting reveals the heterogeneity in tumoral selection of resistance to RAF inhibitors. Cancer Res 73 : 7101-7110, 2013.

27. Sui X, Kong N, Ye L, Han W, Zhou J, Zhang Q, He C and Pan H: p38 and JNK MAPK pathways control the balance of apoptosis and autophagy in response to chemotherapeutic agents. Cancer Lett 344: 174-179, 2014.

28. Pritchard AL and Hayward NK: Molecular pathways: Mitogenactivated protein kinase pathway mutations and drug resistance. Clin Cancer Res 19: 2301-2309, 2013.

29. Reddy P, Liu L, Ren C, Lindgren P, Boman K, Shen Y, Lundin E, Ottander U, Rytinki M and Liu K: Formation of E-cadherinmediated cell-cell adhesion activates AKT and mitogen activated protein kinase via phosphatidylinositol 3 kinase and ligandindependent activation of epidermal growth factor receptor in ovarian cancer cells. Mol Endocrinol 19: 2564-2578, 2005.

30. Tsai MS, Weng SH, Chen HJ, Chiu YF, Huang YC, Tseng SC, Kuo YH and Lin YW: Inhibition of p38 MAPK-dependent excision repair cross-complementing 1 expression decreases the DNA repair capacity to sensitize lung cancer cells to etoposide. Mol Cancer Ther 11: 561-571, 2012.
31. Csibi A, Fendt SM, Li C, Poulogiannis G, Choo AY, Chapski DJ, Jeong SM, Dempsey JM, Parkhitko A, Morrison T, et al: The mTORC1 pathway stimulates glutamine metabolism and cell proliferation by repressing SIRT4. Cell 153: 840-854, 2013

32. Siegel R, Naishadham D and Jemal A: Cancer statistics, 2013. CA Cancer J Clin 63: 11-30, 2013.

33. Center MM, Jemal A, Smith RA and Ward E: Worldwide variations in colorectal cancer. CA Cancer J Clin 59: 366-378, 2009.

34. Härmä V, Knuuttila M, Virtanen J, Mirtti T, Kohonen P, Kovanen P, Happonen A, Kaewphan S, Ahonen I, Kallioniemi O, et al: Lysophosphatidic acid and sphingosine-1-phosphate promote morphogenesis and block invasion of prostate cancer cells in three-dimensional organotypic models. Oncogene 31: 2075-2089, 2012

35. Wu XX, Kakehi Y, Mizutani Y, Lu J, Terachi T and Ogawa O: Activation of caspase-3 in renal cell carcinoma cells by anthracyclines or 5-fluorouracil. Int J Oncol 19: 19-24, 2001.

36. Samuel T, Fadlalla K, Gales DN, Putcha BD and Manne U: Variable NF- $\kappa \mathrm{B}$ pathway responses in colon cancer cells treated with chemotherapeutic drugs. BMC Cancer 14: 599, 2014.

37. Shi X, Wu S, Yang Y, Tang L, Wang Y, Dong J, Lü B, Jiang G and Zhao W: AQP5 silencing suppresses p38 MAPK signaling and improves drug resistance in colon cancer cells. Tumour Biol 35: 7035-7045, 2014

38. Jeong S, Jing K, Kim N, Shin S, Kim S, Song KS, Heo JY, Park JH, Seo KS, Han J, et al: Docosahexaenoic acid-induced apoptosis is mediated by activation of mitogen-activated protein kinases in human cancer cells. BMC Cancer 14: 481, 2014. 